\title{
Immuno disc assay for screening duck hepatitis B surface antigen in serum, liver tissue and cultured hepatocytes
}

\author{
G.A. de Wilde and R.A. Heijtink \\ Department of Virology, Erasmus University, Rotterdam (The Netherlands) \\ (Accepted 16 December 1992)
}

\section{Summary}

An immuno disc assay (IDA) for semi-quantitative analysis of the surface antigen (DHBsAg) of duck hepatitis B virus (DHBV) is described. Unpurified antigen preparations were adsorbed onto punched-out nitrocellulose membrane discs. Rabbit antiserum raised against serum-derived gradient-purified DHBsAg was used for detecting the antigen. Cross-reacting antibodies in the rabbit antiserum were removed using normal duck serum and normal duck hepatocytes. The sensitivity of the IDA was compared with that of the Western blot analysis and was observed to be of the same order, but differed slightly for DHBsAg in liver and sera. In contrast to Western blot analysis, antigen specificity for the IDA included the $S$-protein. Immunodetection was carried out in microtitre plates, but the procedure was accelerated by attaching the antigen-adsorbed discs to an adhesive plate sealer. The IDA was exemplified for measuring DHBsAg in duck serum, duck liver homogenates and viral protein synthesis in cultures of DHBV-infected hepatocytes.

Immunoassay; Western blotting; DHBV; DHBsAg; Serum; Hepatocytes

\section{Introduction}

The Pekin duck is the natural host for the duck hepatitis B virus (DHBV) which belongs to the family of Hepadnaviridae (Mason et al., 1980). The 
genome of these viruses contains four major open reading frames, namely $\mathrm{S}, \mathrm{C}$, $\mathrm{P}$, and $\mathrm{X}$. The $\mathrm{S}$ open reading frame is further divided into pre-S1, pre-S2 and the $\mathrm{S}$ genes coding for the hepatitis $\mathrm{B}$ surface antigens. The animal model offers a new system for hepatitis B virus (HBV) research (Marion, 1988; Summers, 1981).

As a rule in vivo and in vitro DHBV infections are monitored by DHBV DNA hybridization. Further detailed analysis of the infection process requires additional information on antigen production, similar to that for the human $\mathrm{HBV}$ infection in which HBsAg serves as a serological marker for infection.

In contrast to the detection of human hepatitis B surface antigen ( $\mathrm{HBs} A \mathrm{~A})$, commercial tests are not available for detecting duck hepatitis B surface antigen (DHBsAg) and in the past conventional laboratory techniques such as radioimmunoassay, immunoprecipitation or Western blotting were used (Civitico et al., 1990; Galle et al., 1989; Niu et al., 1990; Qiao et al., 1990; Yokosuka et al., 1988).

In this study, the aim was to use readily available polyclonal antibodies in an assay system which did not require any special equipment and which was suitable for screening unpurified samples from different sources such as whole serum and crude extracts.

A modified immuno dot blot assay for DHBsAg (IDA: immuno disc assay) is described in which antigen-containing samples were passively adsorbed onto nitrocellulose membrane to yield a more homogeneous distribution of the antigen as compared with membrane filtration techniques.

\section{Materials and Methods}

\section{Experimental animals}

Uninfected Pekin ducks (Anas domesticus) were purchased from a commercial supplier (VSE, Harderwijk, The Netherlands) and served as a breeding stock for DHBV-negative animals. A breeding stock with congenital chronic DHBV infection was established by inoculating (intravenously) 1-dayold ducklings with serum with a high titre of DHBV. The DHBV strain was kindly provided by Dr. K.N. Tsiquaye (London, UK).

\section{DHBs Ag antigen samples}

Sera from 6 DHBV-positive and 12 DHBV-negative ducks were collected. Livers obtained from an additional 4 DHBV-positive and 4 DHBV-negative ducks were cut into pieces, snap-frozen in liquid nitrogen and stored at $-70^{\circ} \mathrm{C}$. Cells and supernatants of experimentally infected primary cultures of Pekin duck hepatocytes served as sources of intracellular and extracellular DHBsAg. 


\section{Detection of viral DNA}

The presence of DHBV DNA in serum and liver tissue was demonstrated by dot blot and Southern blot analysis respectively, using ${ }^{32} \mathrm{P}$-labelled (cloned) DHBV DNA according to the standard laboratory techniques (Sambrook et al., 1989). Plasmid pBR322 containing the full length $(3.0 \mathrm{~kb})$ genome of DHBV was kindly provided by Dr. H.E. Blum (Freiburg, Germany).

\section{Preparation of rabbit anti-DHBsAg serum}

Serum from chronically infected Pekin ducks was used as the source of DHBsAg. DHBsAg was purified by gradient centrifugation as described by Heijtink et al. (1989) using a cesium chloride gradicnt $[0-50(\mathrm{w} / \mathrm{w}) \mathrm{CsCl}]$ instead of the potassium chloride gradient. The appropriate DHBsAg containing fractions were pooled and stored after determining their protein composition using polyacrylamide gel electrophoresis (Schlicht et al., 1987).

New Zealand white rabbits were immunized with purified DHBsAg. $10 \mu \mathrm{g}$ DHBsAg was emulsified in Freund's complete adjuvant and injected intracutaneously (i.c.) at four sites. Booster injections (i.c.) with $10 \mu \mathrm{g}$ DHBsAg emulsified in incomplete Freund's adjuvant were given 3 and 5 wk after the initial immunization. Antibody production was monitored by Western blot (WB) analysis using a reference rabbit anti-DHBsAg serum kindly provided by Dr. W.H. Gerlich (Göttingen, Germany). Three weeks after the second booster injection, blood was collected.

The DHBsAg preparation used for producing antibody contained small amounts of normal serum proteins. Antibodies against these proteins were removed by absorption with normal duck serum (NDS) coupled to $\mathrm{CNBr}$ activated Sepharose 4B (Pharmacia Fine Chemicals AB, Uppsala, Sweden). Possible cross-reacting antibodies against normal duck hepatocytes (NDH) were removed by batch-wise absorption with hepatocytes obtained by liver perfusion (see below). Aliquots of the NDS-absorbed rabbit immune serum were repeatedly incubated with glutaraldehyde fixed hepatocytes. The absorbed rabbit immune serum was stored at $-20^{\circ} \mathrm{C}$.

\section{Liver perfusion and hepatocyte cultures}

Pekin duck hepatocytes were obtained from 2-wk-old DHBV-negative ducklings by collagenase perfusion using a Hepes buffered system (Moshage et al., 1988; Seglen, 1976). The cells were seeded onto $75 \mathrm{~cm}^{2}$ flasks (Nunc) and 60 $\mathrm{mm}$ dishes (Primaria, Falcon) at a density of $2 \times 10^{5}$ cells $/ \mathrm{cm}^{2}$ in William's medium E (Flow Laboratories) supplemented with $2 \mathrm{mM}$ L-glutamine, $20 \mathrm{mU} /$ $\mathrm{ml}$ insulin (porcine insulin, Organon, Oss, The Netherlands), $0.05 \mathrm{mg} / \mathrm{ml}$ gentamycin, $0.05 \mathrm{mM}$ hydrocortisone and 1\% (v/v) DMSO (Sigma, tissue culture grade). The cultures were maintained at $37^{\circ} \mathrm{C}$ in $5 \% \mathrm{CO}_{2}$ and $98 \%$ humidity. One day after seeding and subsequently every other day, the medium 
was replaced. Confluent monolayers were established within 2 days.

The cultured hepatocytes were used for absorption of the rabbit immune serum with normal duck hepatocytes (flasks) and for establishing an experimental DHBV infection (dishes).

\section{Experimental $D H B V$ infection in vitro}

Hepatocyte cultures in dishes were inoculated on day 3 after seeding with serial $10^{-0.5}$ dilutions of a DHBV DNA-positive duck serum in culture medium (dilutions $10^{-1.5}-10^{-4.5}$ ). After adsorption for $5 \mathrm{~h}$ at $37^{\circ} \mathrm{C}$ (Pugh and Summers, 1989) the monolayer was washed with PBS and then incubated with fresh culture medium. Culture supernatants were harvested every other day and replaced by fresh medium.

Supernatants from duplicate cultures were pooled. Cells were harvested by scraping and suspended in Tris buffer (TBS: $20 \mathrm{mM}$ Tris $\cdot \mathrm{HCl}, \mathrm{pH} 7.5$, with 0.5 $\mathrm{M} \mathrm{NaCl}$ ).

Sample preparation for IDA and Western blot (WB)

Liver tissue in TBS was homogenized in a Virtis S23 apparatus (The Virtis $\mathrm{Co}$, Gardiner, NY, USA) and the suspension was centrifuged at $12000 \times g$ for $15 \mathrm{~min}$. Total protein concentration of the supernatant was measured using a protein assay (Bio-Rad Laboratories) and adjusted to a final concentration of $20 \mathrm{mg} \operatorname{protein} / \mathrm{ml}$.

Cultured hepatocytes were lysed by freeze-thawing. The lysates were centrifuged at $12000 \times g$ for 5 min. The supernatants were collected.

Culture supernatants were concentrated by PEG precipitation (Kimpton et al., 1990).

Sera were used without further treatment.

$S D S-P A G E$ and $W B$ analysis

SDS-PAGE, Western blotting and immunodetection were performed as described previously (Heijtink et al., 1989). Serial 2-fold dilutions of serum and liver homogenates were subjected to SDS polyacrylamide gel electrophoresis followed by Western blotting. Rabbit anti-DHBsAg (absorbed) and horse antirabbit Ig (CLB, Amsterdam, The Netherlands) were used for immunodetection.

Immuno disc assay (IDA)

A nitrocellulose (NC) membrane with $0.10-\mu \mathrm{m}$ pores (Schleicher and Schuell) was attached to an adhesive plate sealer (Flow Laboratories) and 5$\mathrm{mm}$ discs were punched-out with a perforator. The discs were placed in the wells of a flat bottom 96-well tissue culture plate (Greiner) with the 
nitrocellulose side up. Two-fold dilutions of the samples in TBS were prepared in round-bottom microtitre plates (Sterilin) and $100 \mu \mathrm{l}$ of each dilution were transferred to the flat-bottom plate containing the discs and incubated overnight. All incubations were at room temperature under continuous shaking. The unadsorbed material was removed by aspiration and rinsing three times with TBS. Subsequently, the discs were either dried (at least $30 \mathrm{~min}$ at $37^{\circ} \mathrm{C}$ ) and temporarily stored (dry!) in the wells of the plates at $-20^{\circ} \mathrm{C}$ or stained immediately. After drying, the discs had to be wetted with $100 \mu \mathrm{l}$ TTBS (TBS $+0.05 \%(\mathrm{v} / \mathrm{v})$ Tween 20$)$ per well for 30 min prior to blocking.

Initially immunostaining was carried out in the wells of the plate (procedure I). In a later phase (procedure II), the dry (antigen-adsorbed) discs were attached with the nitrocellulose side up to an adhesive plate sealer which facilitated and accelerated the procedure becausc the discs formed one sheet which could be handled as a single membrane. Buffer and incubation conditions were the same as for procedure I. The buffer volume was approximately $1 \mathrm{ml} / \mathrm{cm}^{2}$. In procedure $\mathrm{I}$, the discs were blocked by incubation with $100 \mu \mathrm{l} /$ well blocking medium (BM) consisting of TBS $+5 \%(\mathrm{w} / \mathrm{v})$ non-fat dried milk (Protifar, Nutricia, Zoetermeer, The Netherlands) and $0.01 \%(\mathrm{v} / \mathrm{v})$ anti-foam (Sigma) for $1 \mathrm{~h}$. The BM was then removed and replaced with $50 \mu \mathrm{l}$ BM containing 4\% (v/v) normal goat serum (Zymed) and 30 min later, $50 \mu \mathrm{l}$ rabbit anti-DHBsAg (absorbed) immune serum in BM (final dilution 1:200) were added. After an overnight incubation, the discs were rinsed three times for 5 min with $100 \mu \mathrm{l}$ TTBS and $100 \mu$ l horse anti-rabbit Ig (CLB) conjugated with HRP in BM (optimal dilution 1:500) were added to the wells and incubated for $2 \mathrm{~h}$. Subsequently, the discs were rinsed, as before, and rinsed additionally with TBS for $10 \mathrm{~min}$. Substrate containing $\alpha$-chloro-1-naphthol (HRP Color Development Reagent, Bio-Rad Laboratories) was added in a volume of 150 $\mu \mathrm{l} /$ well until maximum colour developed (after approximately $30 \mathrm{~min}$ ). The reaction was stopped by rinsing the discs with distilled water. End-point titres were read visually. Purified DHBsAg served as an internal standard.

\section{Results}

Characterization of the rabbit anti-DHBsAg immune serum

The purity of the DHBsAg preparation was assessed by SDS-PAGE and silver staining. A representative pattern of the main viral-specific polypeptide bands: S (17 kDa), pre-S2 $(27 \mathrm{kDa})$ and pre-S1 $(36 \mathrm{kDa})$ can be seen in Fig. 1 (lane 2) (Schlicht et al., 1987; Yokosuka et al., 1988).

Western blotting followed by immune staining showed a range $(25-38 \mathrm{kDa})$ of polypeptides at the pre-S locations (Fig. 1, lane 3). In agreement with Yokosuka et al. (1988), no staining was observed at the location of the $\mathrm{S}$ polypeptide because of the denaturing and reduction conditions that were required for SDS-PAGE and the quality of the antibodies that were evoked in 


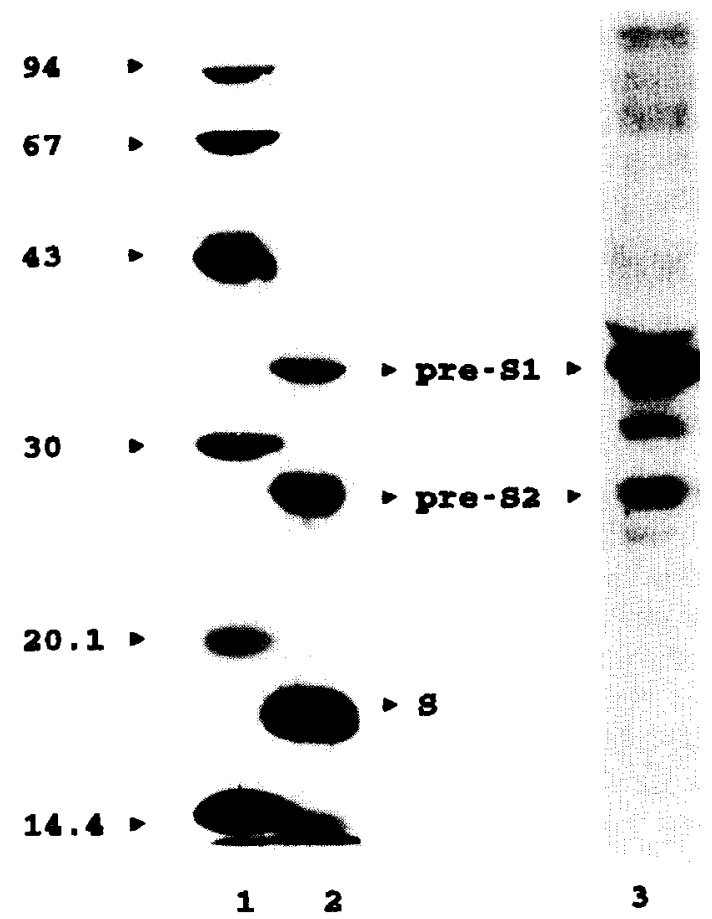

Fig. 1. Characteristic pattern of serum-derived gradient-purified DHBsAg. Silver-stained polyacrylamide gel: low molecular weight markers in $\mathrm{kDa}$ (Pharmacia) and DHBsAg in lanes 1 and 2, respectively. WB analysis of DHBsAg using rabbit anti-DHBsAg (absorbed) in lane 3.

the rabbit. It is assumed that the immune response is directed largely against conformational epitopes, as was observed for the $\mathrm{S}$ protein of human hepatitis B virus (Heerman et al., 1987).

In the IDA, both conformational and linear epitopes of DHBsAg are involved in the reaction with the rabbit immune serum. This was demonstrated

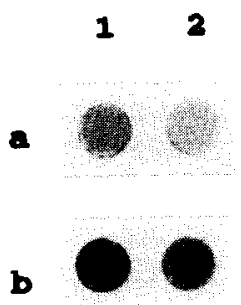

Fig. 2. IDA of two DHBsAg-containing sera incubated in the absence or presence of 2-mercaptoethanol. Columns 1 and 2: discs incubated with the two sera (undiluted). After rinsing and drying, the discs were incubated in TBS for $2 \mathrm{~h}$ at $56^{\circ} \mathrm{C}$ with (row a) or without (row b) $5 \%$ (v/v) 2-mercaptoethanol. Immunodetection procedure II. 
A

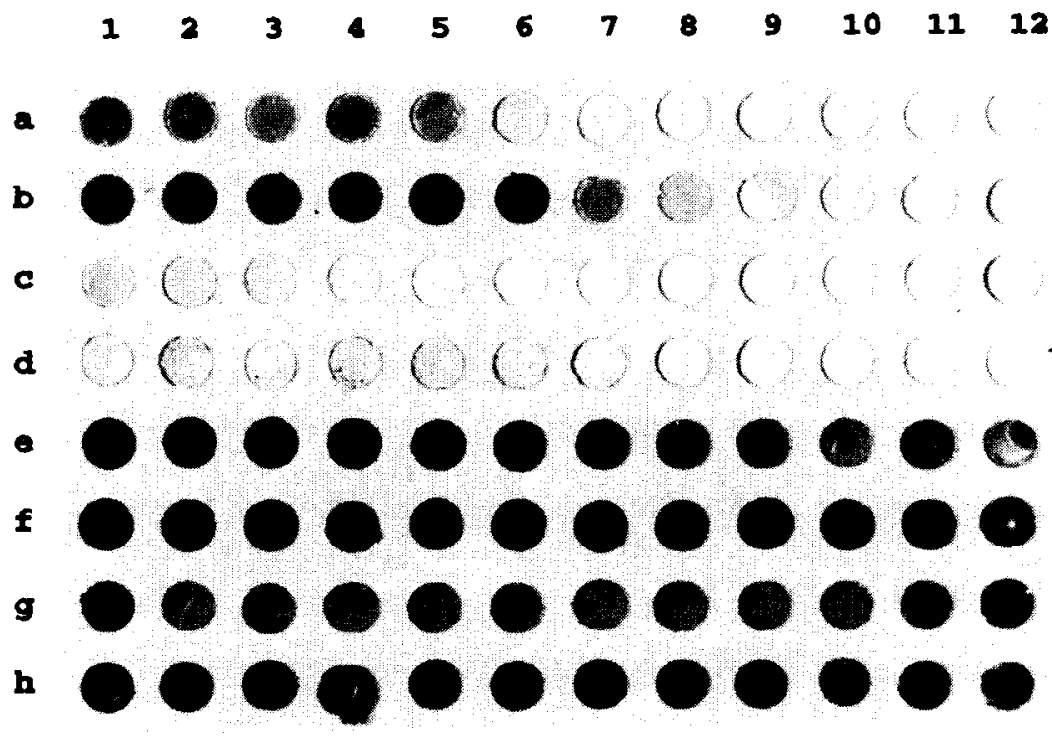

B

$\begin{array}{llllllllllll}1 & 2 & 3 & 4 & 5 & 6 & 7 & 8 & 9 & 10 & 11 & 12\end{array}$

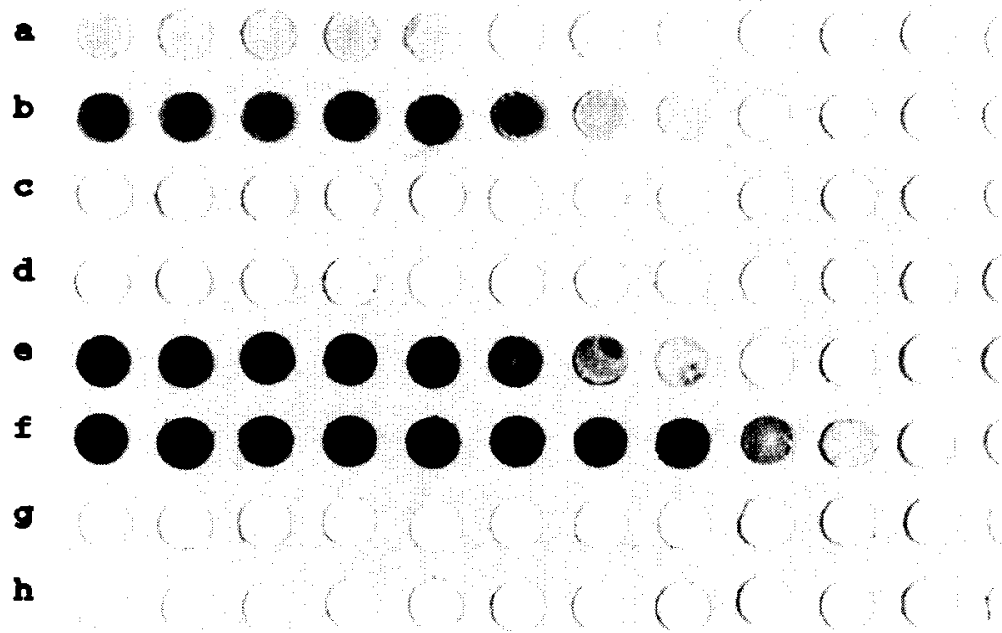

Fig. 3. Specificity of the rabbit anti-DHBsAg immune serum in the disc immunobinding assay (IDA): antiDHBsAg (unabsorbed) (A) and anti-DHBsAg (absorbed) (B). Undiluted samples and 2-fold dilutions of four liver homogenates (DHBV-positive: rows a, b; DHBV-negative: rows c, d) and four sera (DHBVpositive: rows e, f; DHBV-negative: rows $\mathrm{g}, \mathrm{h}$ ). Immunodetection procedure II. 
by incubating DHBsAg-positive serum adsorbed onto nitrocellulose discs in TBS, with or without $5 \%(\mathrm{v} / \mathrm{v}) 2$-mercaptoethanol treatment for $2 \mathrm{~h}$ at $56^{\circ} \mathrm{C}$, and subsequent immunostaining. A decreased staining intensity was noted in the presence of 2-mercaptoethanol which could not be explained by loss of the protein from the discs (Fig. 2). The latter was checked by staining (Hancock and Tsang, 1983) the antigen loaded discs treated with and without 2mercaptoethanol with India ink.

Specificity of antiserum after absorption

The specificity of the rabbit anti-DHBsAg serum after absorption was confirmed by assessing the DHBsAg content of DHBV-positive and negative sera and liver homogenates using the IDA. Both absorbed and unabsorbed immune sera were used as primary antibody (Figs. 3A and B). A clear distinction between stained (DHBsAg-positive) and unstained (DHBsAgnegative) discs was only observed when absorbed immune serum was used. Background staining was absent.

Evaluation of the immuno disc assay (IDA) by comparison with $W B$ analysis

The relative sensitivities of the IDA and WB analysis for total DHBsAg and pre-S, respectively, were determined by using serial dilutions of serum and liver homogenates. Figs. 4A and B show that end-point titres by IDA (DHBsAg) were similar for the liver homogenates and slightly higher $(\geqslant 4)$ for the sera than those determined by WB analysis (reading pre-S1). Samples from uninfected animals were negative (results not shown).

Application of the immuno disc assay (IDA) in an infectivity study

DHBV DNA-positive serum was used to produce an experimental infection in a primary culture of Pekin duck hepatocytes in a dose-response experiment (Fig. 5). On day 8 after infection, extracellular DHBsAg was observed with the $10^{-1.5}$ inoculum preparation. As the time increased, a positive signal was observed at higher dilutions.

On day 14 after infection, intracellular and extracellular DHBsAg production was ultimately established with an inoculum diluted $10^{-4.0}$ and $10^{-3.5}$, respectively.

Using the same DHBV-positive serum (dilution of $10^{-1.5}$ ), starting on day 6 the intracellular DHBV DNA was confirmed using Southern blot hybridization (results not shown).

\section{Discussion}

A simple and multi-purpose DHBsAg immuno disc assay (IDA) is described. 


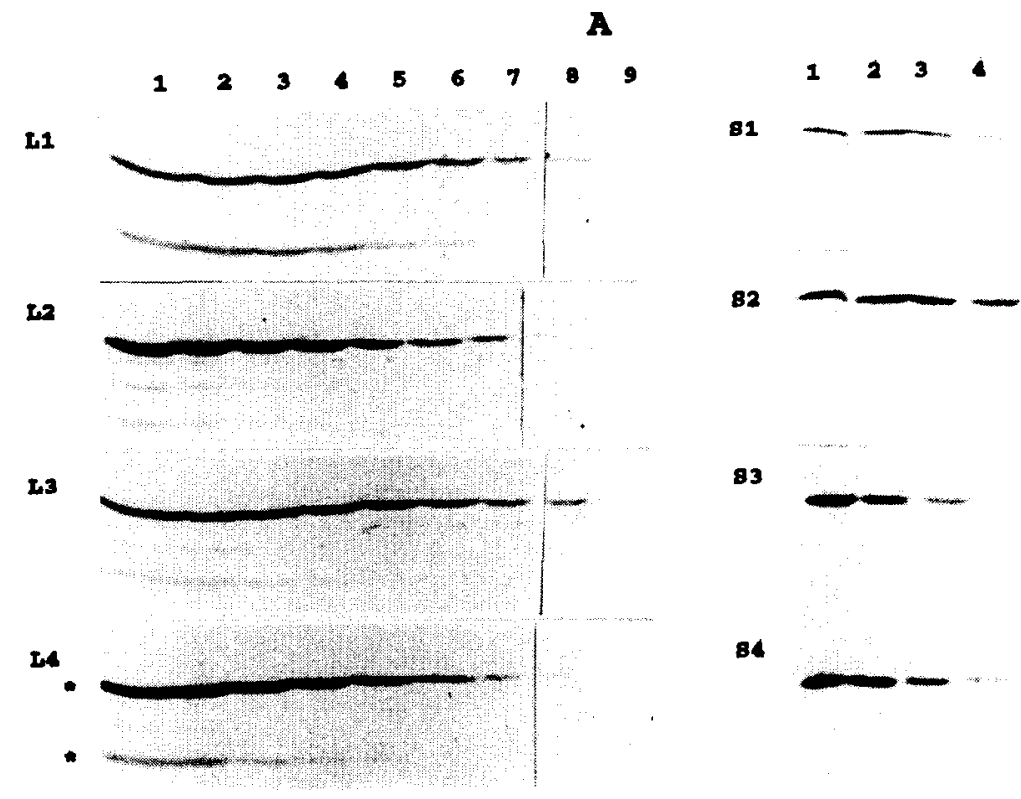

B
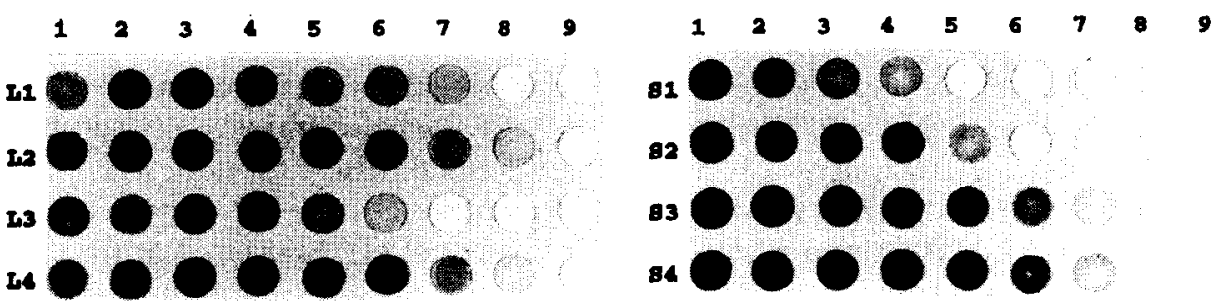

Fig. 4. Relative sensitivities of the IDA and the WB analysis for DHBsAg based on end-point titration of four DHBV-positive liver homogenates (L1-L4) and four DHBV-positive sera (S1-S4). WB analysis (A): undiluted (1/1) samples and/or 2-fold dilutions (lanes 1-9) L1:1/2-1/512; L2:1/8-1/2048; L3:1/2-1/512; L4:1/4-1/1024; (lanes 1-6) S1:1/1-1/32; S2:1/1-1/32; S3:1/8-1/256; S4:1/4-1/128. An asterisk (*) indicates the location of the pre-S1 and pre-S2 polypeptides (L4 as an example). IDA (B): 2-fold dilutions of L1-L4 and S1-S4 1/8-1/2048 (columns 1-9). Immunodetection procedure I.

The IDA is based on the adsorption of protein (unpurified/non-denatured antigen) to nitrocellulose discs, and immunodetection of the antigen by a method similar to that of Western blot analysis. The specificity of the IDA was guaranteed by using a specific rabbit (anti-DIIBsAg) immune serum which was freed of antibodies against non-viral proteins by absorption with normal duck serum and normal duck hepatocytes. The high specificity of the IDA indicates that antigens from several sources may be assessed, broadening its applicability.

Optimum antigen adsorption was obtained by using a nitrocellulose membrane (NC) with $0.10-\mu \mathrm{m}$ pores instead of the commonly used $0.45-\mu \mathrm{m}$ 


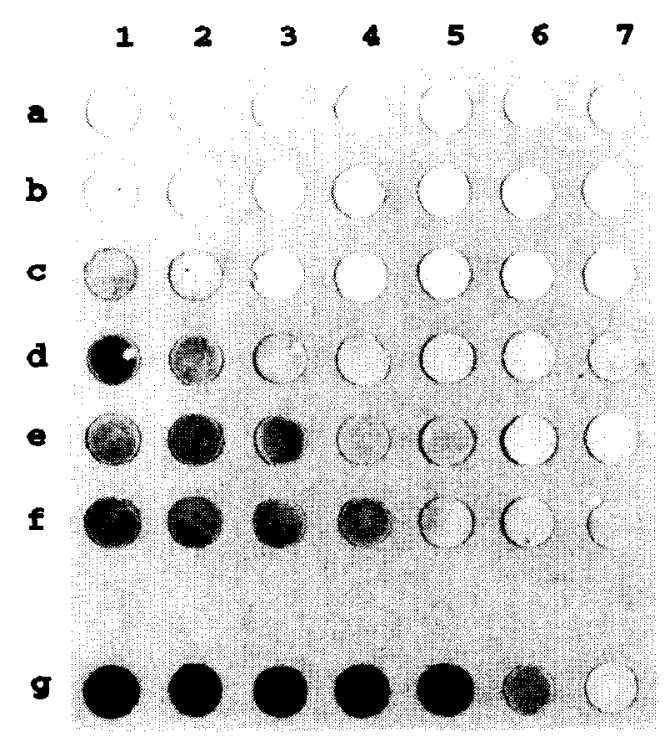

Fig. 5. Application of the IDA in a dose-response study of an in vitro DHBV infection. Columns 1-7 correspond to the harvest from hepatocyte cultures inoculated with $10^{-0.5}$ fold dilutions $\left(10^{-1.5}-10^{-4.5}\right)$ of a DHBV-positive serum. Rows a-f: extracellular DHBsAg 4, 6, 8, 10, 12 and 14 days after infection. Row $\mathrm{g}$ : intracellular $\mathrm{DHBs} \Lambda \mathrm{g} 14$ days after infection. Immunodetection procedure $\mathbf{I}$.

pores. The adhesive tape backing made the NC discs easy to handle. The microtitre system allowed the processing of large numbers of samples simultaneously. Attachment of the antigen-loaded discs to adhesive plate sealers accelerated the immunodetection procedure.

It was assumed (Heerman et al., 1987; Yokosuka et al., 1988) that antibodies against the S protein of (D)HBV, evoked by immunizing rabbits with native viral particles, were directed largely against conformational epitopes. In our studies, using rabbit immune serum against native viral particles, we were unable to demonstrate the denatured and reduced S-protein by WB analysis. In the IDA, however, we observed a diminishing immune reaction against DHBsAg adsorbed onto nitrocellulose discs after treatment with 2-mercaptoethanol indicating the sustained presence of conformational epitopes. For this reason the end-point titres obtained with IDA and WB analysis cannot be strictly compared, but both indicate that DHBV-specific proteins are produced during the infection.

In summary, with the immunobinding disc assay for measuring DHBsAg in serum, liver tissue and hepatocyte cultures, non-denatured proteins of DHBV are detected. IDA is less laborious than WB analysis, requires minimal laboratory facilities and is applicable to both in vivo and in vitro studies. It can be used to monitor experimental DHBV infections and to assess the effect of antiviral agents on DHBV protein synthesis. 


\section{Acknowledgements}

The authors are grateful to Dr. W.J. Kort, Mrs. J.M. Hekking-Weyma and Mr. J. Kasbergen at the laboratory for experimental surgery for the excellent technical assistance with the ducks and to Dr F.W.M. de Rooy for reading critically the manuscript.

\section{References}

Civitico, G., Wang, Y., Luscombe, C., Bishop, N., Tachedjian, G., Gust, I. and Locarni, S. (1990) Antiviral strategies in chronic hepatitis B virus infection: II. Inhibition of duck hepatitis B virus in vitro using conventional antiviral agents and supercoiled-DNA active compounds. J. Med. Virol. 31, 90-97.

Galle, P.R., Schlicht, H-J., Kuhn, C. and Schaller, H. (1989) Replication of duck hepatitis B virus in primary duck hepatocytes and its dependence on the state of differentiation of the host cell. Hepatology 10, 459-465.

Hancock, K. and Tsang, V.C.W. (1983) India ink staining of proteins on nitrocellulose paper. Anal. Biochem. 133, 157-162.

Heerman, K.H., Kruse, F., Seifer, M. and Gerlich, W.H. (1987) Immunogenicity of the gene S and pre-S domains in hepatitis $B$ virions and HBsAg filaments. Intervirol. 28, 14-25.

Heijtink, R.A., de Wilde, G.A., van Hattum, J. and Schalm, S.W. (1989) Long-term immune reactivity to pre-S(2)-antigen after acute hepatitis B infection. J. Med. Virol. 27, 95-99.

Kimpton, C.P., Corbitt, G. and Morris, D.J. (1990) Comparison of polyethylene glycol precipitation and ultracentrifugation for recovery of cytomegalovirus from urine prior to detection of DNA by dot-blot hybridisation. J. Virol. Methods 28, 141-146.

Marion, P.L. (1988) Use of animal models to study hepatitis B virus. Prog. Med. Virol. 35, 43-75.

Mason, W.S., Seal, G. and Summers, J. (1980) Virus of Pekin ducks with structural and biological relatedness to human hepatitis B virus. J. Virol. 36, 829-836.

Moshage, H.J., Rijntjes, P.J.M., Hafkenscheid, J.C.M., Roelofs, H.M.J. and Yap, S.H. (1988) Primary culture of cryopreserved adult human hepatocytes on homologous extracellular matrix and the influence of monocytic products on albumin synthesis. J. Hepatol. 7, 3444.

Niu, J., Wang, Y., Qiao, M., Gowans, E., Edwards, P., Thyagarajan, S.P., Gust, I. and Locarnini, S. (1990) Effect of Phyllanthus amarus on duck hepatitis B virus replication in vivo. J. Med. Virol. $32,212-218$.

Pugh, J.C. and Summers, J.W. (1989) Infection and uptake of duck hepatitis B virus by duck hepatocytes maintained in the presence of dimethyl sulfoxide. Virology 172, 564-572.

Qiao, M., Gowans, E.J., Bailey, S.E., Jilbert, A.R. and Burrell, C.J. (1990) Serological analyses of duck hepatitis B virus infection. Virus Res. 17, 3-14.

Sambrook, J., Fritsch, E.F. and Maniatis, T. (1989) Molecular Cloning: A Laboratory Manual, 2nd ed. Cold Spring Harbor Laboratory, Cold Spring Harbor, New York.

Schlicht, H.J., Kuhn, C., Guhr, B., Mattaliano, R.J. and Schaller, H. (1987) Biochemical and immunological characterization of the duck hepatitis B virus envelope proteins. J. Virol. 61, 2280-2285.

Seglen, P.O. (1976) Preparation of isolated rat liver cells. In: Prescott, D.M. (Ed), Methods in Cell Biology, Vol. 13, Academic Press, New York, pp. 29-83.

Summers, J. (1981) Three recently described animal virus models for human hepatitis B virus. Hepatology 1, 179-183.

Yokosuka, O., Omata, M. and Ito, Y. (1988) Expression of pre-S1, pre-S2, and C proteins in duck hepatitis B infection. Virology $167,82-86$. 